\title{
FIXED POINT THEOREMS FOR DENSIFYING MAPPINGS AND COMPACT MAPPINGS
}

\author{
ZEQING LIU, LI WANG, SHIN MIN KANG, and YONG SOO KIM
}

Received 8 April 2002

\begin{abstract}
The purpose of this note is to establish fixed point theorems for densifying mappings and compact mappings which are contractive in metric spaces and to investigate the existence of fixed points for a family of mappings in bounded metric spaces. The results of this note generalize the results of Bailey (1966) and Liu (1994).
\end{abstract}

2000 Mathematics Subject Classification: 54H25.

1. Introduction and preliminaries. Let $f$ be a self-mapping of a metric space $(X, d)$, $N$ denote the positive integers set, and $N_{0}=N \cup\{0\}$. Contractive mappings in metric spaces have been interested for many years, one of those is

$$
d\left(f^{n(x, y)} x, f^{n(x, y)} y\right)<d(x, y)
$$

for all distinct $x, y \in X$, where $n(x, y) \in N$. Bailey [1] has investigated the existence of fixed points for the contractive mapping (1.1) in compact metric spaces. Liu [4] first introduced the definition of the family of mappings $\mathrm{CIS}_{f}$, and showed fixed point theorems for $\mathrm{CIS}_{f}$. In this note, we prove a few fixed point theorems for densifying and compact mappings which satisfy (1.1) in complete metric spaces and metric spaces, respectively. We also give an example to show that our results are the proper generalizations of the result of Bailey [1]. On the other hand, we go on investigating the existence of fixed points for $\mathrm{CIS}_{f}$ in bounded metric spaces, and our results extend the result of Liu [4].

The following definitions were introduced by Bailey [1] and Nussbaum [5].

DEFINITION 1.1 (see [1]). For $x, y \in X, x$ is proximal to $y$ under $f$ provided that for each $\varepsilon>0$ there exists $n \in N$ such that $d\left(f^{n} x, f^{n} y\right)<\varepsilon$. If $x$ and $y$ are not proximal, they are said to be distal.

Definition 1.2 (see [5]). A nonempty subset $M$ of $X$ is said to be an attractor for compact sets under $f$ if (1) $M$ is compact and $f(M) \subseteq M$, and (2) given any compact set $C \subseteq X$ and any open neighborhood $U$ of $M$, there exists $k \in N$ such that $f^{n}(C) \subseteq U$ for $n \geq k$.

For $S \subseteq X, \delta(S)$ and $\bar{S}$ denote the diameter and the closure of $S$, respectively. For $A, B \subseteq X, \delta(A, B)=\sup \{d(x, y) \mid x \in A, y \in B\}$. Following Furi and Vignoli [2], $f$ is said to be densifying if for every bounded subset $A$ of $X$ with $\alpha(A)>0$, we have $\alpha(f(A))<\alpha(A)$, where $\alpha(A)$ denotes the measure of noncompactness in the sense 
of Kuratowski. The mapping $f$ is said to be compact if there exists a compact subset $Y$ of $X$ such that $f(X) \subseteq Y$. It is well known that every continuous compact mapping is a densifying mapping. Introduced by Liu [4], CIS ${ }_{f}=\{h: X \rightarrow X \mid$ for every nonempty compact $f$-invariant subset $A$ of $h(A) \subseteq A\}$. Let $O(x, f)=\left\{f^{n} x \mid\right.$ $\left.n \in N_{0}\right\}$ and $O(x, y, f)=O(x, f) \cup O(y, f)$, for $x, y \in X$, where $f^{0}$ is the identity mapping in $X$. The mapping $f$ is said to have diminishing orbital diameters if $\lim _{n \rightarrow \infty} \delta\left(O\left(f^{n} x, f\right)\right)<\delta(O(x, f))$ for all $x \in X$ with $0<\delta(O(x, f))<\infty$.

\section{Main results}

THEOREM 2.1. Let $(X, d)$ be a complete metric space and $f: X \rightarrow X$ be a continuous densifying mapping satisfying (1.1). Suppose that $O\left(x_{0}, f\right)$ is bounded for some $x_{0} \in X$. Then $f$ has a unique fixed point in $X$.

Proof. Since $f$ is densifying and

$$
\alpha\left(O\left(x_{0}, f\right)\right)=\max \left\{\alpha\left(f\left(O\left(x_{0}, f\right)\right)\right), \alpha\left(x_{0}\right)\right\}=\alpha\left(f\left(O\left(x_{0}, f\right)\right)\right),
$$

it follows that $\alpha\left(O\left(x_{0}, f\right)\right)=0$. From the completeness of $(X, d)$, we know that $\overline{O\left(x_{0}, f\right)}$ is compact in $X$. We claim that $x_{0}$ and $f x_{0}$ are proximal under $f$. If $f^{n} x_{0}=f^{n+1} x_{0}$ for some $n \in N$, then $x_{0}$ and $f x_{0}$ are proximal under $f$. If $f^{n} x_{0} \neq f^{n+1} x_{0}$ for any $n \in N$, from (1.1) we infer that there exists a sequence $\left\{n_{i}\right\}_{i \in N} \subset N$ such that

$$
\begin{aligned}
d\left(x_{0}, f x_{0}\right) & >d\left(f^{n_{1}} x_{0}, f^{n_{1}+1} x_{0}\right) \\
& >d\left(f^{n_{2}} x_{0}, f^{n_{2}+1} x_{0}\right)>\cdots>d\left(f^{n_{i}} x_{0}, f^{n_{i}+1} x_{0}\right)>\cdots
\end{aligned}
$$

for all $i \in N$. Suppose that each $n_{i}$ is chosen as the smallest positive integer in order to satisfy (2.2). Then for any $k \in N$ there exists $n_{i} \in N$ such that $n_{i-1} \leq k<n_{i}$. It follows from (2.2) that

$$
d\left(f^{k} x_{0}, f^{k+1} x_{0}\right) \geq d\left(f^{n_{i-1}} x_{0}, f^{n_{i-1}+1} x_{0}\right)>d\left(f^{n_{i}} x_{0}, f^{n_{i}+1} x_{0}\right) .
$$

Since $\overline{O\left(x_{0}, f\right)}$ is compact and $f$ is continuous, we may (by selecting a subsequence, if necessary) assume that $f^{n_{i}} x_{0} \rightarrow u$ and $f^{n_{i}+1} x_{0} \rightarrow f u$ for some $u \in X$ as $i \rightarrow \infty$. Thus $d(u, f u)=\lim _{i \rightarrow \infty} d\left(f^{n_{i}} x_{0}, f^{n_{i}+1} x_{0}\right)$. Now assume that $x_{0}$ and $f x_{0}$ are distal. Then there exists $\varepsilon_{0}>0$ satisfying $d\left(f^{m} x_{0}, f^{m+1} x_{0}\right) \geq \varepsilon_{0}$ for all $m \in N$. It is easy to see that $n_{i}+k \leq n_{k}$ for every $k$. In view of (2.3) we get that

$$
\begin{aligned}
d\left(f^{k} u, f^{k+1} u\right) & =\lim _{i \rightarrow \infty} d\left(f^{n_{i}+k} x_{0}, f^{n_{i}+k+1} x_{0}\right) \\
& \geq \lim _{i \rightarrow \infty} d\left(f^{n_{i+k}} x_{0}, f^{n_{i+k}+1} x_{0}\right) \\
& =d(u, f u),
\end{aligned}
$$

which is a contradiction to (1.1). Hence $x_{0}$ and $f x_{0}$ are proximal under $f$.

Next, we assert that $f$ has a fixed point in $X$. Without loss of generality, we assume that $f^{n} x_{0} \neq f^{n+1} x_{0}$, for all $n \in N_{0}$. Choose $\left\{n_{j}\right\}_{j \in N_{0}} \subset N$ such that $n_{j}<n_{j+1}$ and

$$
d\left(f^{n_{j}} x_{0}, f^{n_{j}+1} x_{0}\right)<\min \left\{\frac{1}{j}, d\left(f^{n_{j-1}} x_{0}, f^{n_{j-1}+1} x_{0}\right)\right\}
$$


for all $j \in N$, where $n_{0}=0$. It follows from the compactness of $\overline{O\left(x_{0}, f\right)}$ and the continuity of $f$ that there exists $w \in X$ and the subsequence $\left\{f^{n_{j_{i}}} x_{0}\right\}_{i \in N}$ such that $f^{n_{j_{i}}} x_{0} \rightarrow w$ and $f^{n_{j_{i}+1}} x_{0} \rightarrow f w$ as $i \rightarrow \infty$. Replacing $j$ by $j_{i}$ in (2.5) and letting $i \rightarrow \infty$, we deduce that $d(w, f w) \leq \min \{0, d(w, f w)\}=0$. That is, $w=f w$.

To prove the uniqueness of the fixed point of $f$, we assume that $f$ has another fixed point $b \in X$ with $b \neq w$. From (1.1) we infer that

$$
d(w, b)=d\left(f^{n(w, b)} w, f^{n(w, b)} b\right)<d(w, b),
$$

which is impossible. This completes the proof.

The next theorem follows from Theorem 2.1.

THEOREM 2.2. Let $(X, d)$ be a metric space and $f: X \rightarrow X$ be a continuous and compact mapping satisfying (1.1). Then $f$ has a unique fixed point in $X$.

REMARK 2.3. The following example shows that Theorems 2.1 and 2.2 are the proper generalizations of [1, Corollary 2].

EXAMPLE 2.4. Let $X=[0, \infty)$ with the usual metric and define $f: X \rightarrow X$ by $f x=$ $|\sin x|$ for all $x \in X$. Choose $n(x, y)=1$ for all $x, y \in X$ with $0<|x-y| \leq 2$ and $n(x, y)=2$ for all $x, y \in X$ with $|x-y|>2$. It is easy to verify that the conditions of Theorems 2.1 and 2.2 are satisfied and $f$ has a unique fixed point $0 \in X$. But [1, Corollary 2] is not applicable since $X$ is not compact.

THEOREM 2.5. Let $(X, d)$ be a bounded complete metric space and let $f: X \rightarrow X$ be a continuous mapping. Suppose that there exist $p, q, r \in N$ such that $f^{r}$ is densifying and

$$
d\left(f^{p} x, f^{q} y\right)<\delta\left(\left\{\cup_{h \in \mathrm{CIS}_{f}} O(z, h): z \in O(x, y, f)\right\}\right)
$$

for all $x, y \in X$ with $f^{p} x \neq f^{q} y$. Then, we have the following:

(i) $f$ has a unique fixed point $v \in X$ such that $f^{n} x \rightarrow v$ for every $x \in X$;

(ii) $f$ has diminishing orbital diameters;

(iii) for every nonempty compact $f$-invariant subset $Y$ of $X, \cap_{n \in N_{0}} f^{n}(Y)=\{v\}$;

(iv) there exists a bounded complete metric $d^{*}$ on $X$ which is equivalent to $d$ such that $f$ is contractive with respect to $d^{*}$, that is, $d^{*}(f x, f y)<d^{*}(x, y)$ for all $x, y \in X$ with $x \neq y$;

(v) $\mathrm{CIS}_{f}$ has a unique fixed point $v \in X$.

Proof. Let $x$ be an arbitrary element in $X$ and $A=O(x, f)$. Then,

$$
\alpha(A)=\max \left\{\alpha\left(\left\{x, f x, \ldots, f^{r-1} x\right\}\right), \alpha\left(f^{r}(O(x, f))\right)\right\}=\alpha\left(f^{r} A\right) .
$$

Since $f^{r}$ is densifying, $A$ is precompact. It follows from the completeness of $(X, d)$ that $\bar{A}$ is compact. By the continuity of $f$, we conclude that $f(\bar{A}) \subseteq \overline{f(A)} \subseteq \bar{A}$. Thus $\bar{A}$ is $f$-invariant. Set $D=\cap_{n \in N} f^{n}(\bar{A})$. It is well known that $D$ is a nonempty compact subset of $\bar{A}$ and $f(D)=D$. Hence $f^{p}(D)=D, f^{q}(D)=D$. We now assert that $D$ is a singleton. Otherwise there exist two distinct points $u, v \in D$ such that $\delta(D)=d(u, v)$. 
Since $f^{p}(D)=D, f^{q}(D)=D$, there exist $x, y \in D$ such that $f^{p} x=u, f^{q} y=v$ and $f^{p} x \neq f^{q} y$. Obviously, $\delta\left(\left\{\cup_{h \in \mathrm{CIS}_{f}} O(z, h): z \in O(x, y, f)\right\}\right) \subseteq D$. By (2.7) we have

$$
0<\delta(D)=d\left(f^{p} x, f^{q} y\right)<\delta\left(\left\{\cup_{h \in \mathrm{CIS}_{f}} O(z, h): z \in O(x, y, f)\right\}\right) \leq \delta(D),
$$

which is a contradiction. So $D$ is a singleton, say, $D=\{v\}$. Therefore $v$ is a fixed point of $f$.

Next we prove that $v$ is the unique fixed point of $f$. Otherwise $b(\neq v)$ is another fixed point of $f$. From (2.7) we get that

$$
0=d(b, v)<d\left(f^{p} b, f^{q} v\right)<\delta\left(\left\{\cup_{h \in \mathrm{CIS}_{f}} O(z, h): z \in O(b, v, f)\right\}\right) \leq d(b, v),
$$

which is impossible. Hence $v$ is a unique fixed point of $f$. Since $f^{n} x, v \in f^{n}(\bar{A})$, $d\left(f^{n} x, v\right) \leq d\left(f^{n}(\bar{A})\right) \rightarrow 0$ as $n \rightarrow \infty$. That is, $f^{n} x \rightarrow v$ as $n \rightarrow \infty$. Since $(X, d)$ is bounded, for each $x \in X-\{v\}$, we have $0<\delta(O(x, f))<\infty$. In the light of (i), we get that for arbitrary $\varepsilon>0$ there exists $k \in N$ such that $d\left(f^{n} x, v\right)<\varepsilon / 3$ for $n>k$. Consequently,

$$
d\left(f^{i} x, f^{j} x\right) \leq d\left(f^{i} x, v\right)+d\left(f^{j} x, v\right)<\frac{2 \varepsilon}{3}
$$

for all $i, j>k$. It follows that

$$
\delta\left(O\left(f^{n} x, f\right)\right)=\sup \left\{d\left(f^{i} x, f^{j} x\right): i, j \geq n\right\} \leq \frac{2 \varepsilon}{3}<\varepsilon
$$

for $n>k$. This means that $\lim _{n \rightarrow \infty} \delta\left(O\left(f^{n} x, f\right)\right)=0$, so $f$ has diminishing orbital diameters.

Similarly we can show that for every nonempty compact $f$-invariant subset $Y$ of $X$, $\cap_{n \in N_{0}} f^{n}(Y)=\{v\}$.

Now, we prove that (iv) holds. Let $C$ be any nonempty compact subset of $X$. Then

$$
\begin{aligned}
\alpha\left(\cup_{n \in N_{0}} f^{n}(C)\right) & =\max \left\{\alpha\left(\cup_{n=0}^{r-1} f^{n}(C)\right), \alpha\left(f^{r}\left(\cup_{n \in N_{0}} f^{n}(C)\right)\right)\right\} \\
& =\alpha\left(f^{r}\left(\cup_{n \in N_{0}} f^{n}(C)\right)\right) .
\end{aligned}
$$

Let $Y=\overline{\cup_{n \in N_{0}} f^{n}(C)}$. Since $f^{r}$ is densifying and $(X, d)$ is complete, $Y$ is compact and $f(Y) \subseteq \overline{f\left(\cup_{n \in N_{0}} f^{n}(C)\right)} \subseteq Y$. It follows from (iii) that $\cap_{n \in N_{0}} f^{n}(Y)=\{v\}$. This implies that $\delta\left(f^{n}(Y)\right) \rightarrow 0$ as $n \rightarrow \infty$. For every open neighborhood $U$ of $v$, there exists an open ball $B(v, \varepsilon)=\{x \mid x \in X$ and $d(x, v)<\varepsilon\}$ such that $B(v, \varepsilon) \subseteq U$. Since $\lim _{n \rightarrow \infty} \delta\left(f^{n}(Y)\right)=0$, there exists $k \in N$ such that $\delta\left(f^{n}(Y)\right)<\varepsilon$ for $n \geq k$. It follows that $d(x, v) \leq \delta\left(f^{n}(Y)\right)<\varepsilon$ for all $x \in f^{n} Y$. That is, $f^{n}(Y) \subseteq B(v, \varepsilon)$. Hence $f^{n}(C) \subseteq$ $f^{n}(Y) \subseteq B(v, \varepsilon) \subseteq U$ for $n \geq k$. This shows that $\{v\}$ is an attractor for compact sets under $f$. Thus (iv) follows from [3, Theorem and Remark 1].

Finally we conclude that (v) holds. For any $h \in \mathrm{CIS}_{f}$, it follows from (i) and the definition of $\mathrm{CIS}_{f}$ that $h(\{v\}) \subseteq\{v\}$. That is, $h v=v$. Hence $v$ is a fixed point of $\mathrm{CIS}_{f}$. Note that $f \in \mathrm{CIS}_{f}$. By (i), $v$ is the only fixed point of $\mathrm{CIS}_{f}$. This completes the proof. 
REMARK 2.6. By taking $p=q$ in Theorem 2.5, we get the result which improves [4, Theorem 2.1].

TheOrem 2.7. Let $(X, d)$ be a bounded complete metric space and let $f, g: X \rightarrow X$ be continuous and commuting mappings. Suppose that there exist $r, s, p, q \in N$ such that $f^{r}$ and $g^{s}$ are densifying and

$$
d\left(f^{p} x, g^{q} y\right)<\delta\left(\left\{\cup_{h \in \mathrm{CIS}_{f}} O(a, h): a \in O(x, f)\right\} \cup\left\{\cup_{t \in \mathrm{CIS}_{g}} O(b, t): b \in O(y, g)\right\}\right)
$$

for all $x, y \in X$ with $f^{p} x \neq g^{q} y$. Then, we have the following:

(i) $f$ and $g$ have a unique common fixed point $v \in X$ such that $f^{n} x \rightarrow v, g^{n} x \rightarrow v$, for every $x \in X$;

(ii) both $f$ and $g$ have diminishing orbital diameters;

(iii) for every nonempty compact $f$-invariant and $g$-invariant subset $Y$ of $X$, $\cap_{n \in N_{0}} f^{n}(Y)=\{v\}$ and $\cap_{n \in N_{0}} g^{n}(Y)=\{v\}$;

(iv) there exist bounded complete metrics $d^{*}$ and $d^{* *}$ on $X$ which are equivalent to $d$ such that $f$ and $g$ are contractive with respect to $d^{*}$ and $d^{* *}$, respectively, that is $d^{*}(f x, f y)<d^{*}(x, y)$ and $d^{* *}(g x, g y)<d^{* *}(x, y)$ for all $x, y \in X$ with $x \neq y$;

(v) $\mathrm{CIS}_{f}$ and $\mathrm{CIS}_{g}$ have a unique common fixed point $v \in X$.

Proof. For $x, y \in X$, set $A=O(x, f), B=O(y, g), C=\cap_{n \in N} f^{n}(\bar{A}), D=\cap_{n \in N} f^{n}(\bar{B})$. As is the proof of Theorem 2.5 we get that $\bar{A}, \bar{B}$ are nonempty compact and $f$-invariant, $g$-invariant subsets of $X$ with $f(C)=C, g(D)=D$, respectively. Hence $f^{p}(C)=C$, $g^{q}(D)=D$. Suppose that $\delta(C, D)>0$. There exist $u \in C, w \in D$ such that $\delta(C, D)=$ $d(u, w)>0$. From $f^{p}(C)=C, g^{q}(D)=D$, there exist $x \in C, y \in D$ such that $f^{p}(x)=$ $u, g^{q}(y)=w$. Obviously, $\left\{\cup_{h \in \mathrm{CIS}_{f}} O(a, h): a \in O(x, f)\right\} \subseteq C$ and $\left\{\cup_{t \in \mathrm{CIS}_{g}} O(b, t): b \in\right.$ $O(y, g)\} \subseteq D$. According to (2.14) we get

$$
\begin{aligned}
0 & <\delta(C, D)=d\left(f^{p} x, g^{q} y\right) \\
& <\delta\left(\left\{\cup_{h \in \mathrm{CIS}_{f}} O(a, h): a \in O(x, f)\right\} \cup\left\{\cup_{t \in \mathrm{CIS}_{g}} O(b, t): B \in O(y, g)\right\}\right) \\
& \leq \delta(C, D),
\end{aligned}
$$

which is a contradiction. Hence $\delta(C, D)=0$, that is $C=D=\{v\}$ for some $v \in X$. Then $v$ is a common fixed point of $f$ and $g$.

Now, we prove that $f$ and $g$ have the only common fixed point $v \in X$. Otherwise $u$ is a second common fixed point of $f$ and $g$. Using (2.14), we have

$$
\begin{aligned}
0 & <d(u, v)=d\left(f^{p} u, g^{q} v\right) \\
& <\delta\left(\left\{\cup_{h \in \mathrm{CIS}_{f}} O(u, h): a \in O(u, f)\right\} \cup\left\{\cup_{t \in \mathrm{CIS}_{g}} O(v, t): b \in O(v, g)\right\}\right) \\
& \leq \delta(u, v),
\end{aligned}
$$

which is impossible. The rest of the proof goes in a similar fashion as that of Theorem 2.5 , so we omit it. This completes the proof.

REMARK 2.8. By taking $f=g$ and $p=q$ in Theorem 2.7, we obtain the result which generalizes Theorem 2.1 of Liu [4]. 
ACKNOWLEDGMENT. This work was supported by Korea Research Foundation Grant KRF-2001-005-D00002.

\section{REFERENCES}

[1] D. F. Bailey, Some theorems on contractive mappings, J. London Math. Soc. 41 (1966), 101106.

[2] M. Furi and A. Vignoli, A fixed point theorem in complete metric spaces, Boll. Un. Mat. Ital. (4) 2 (1969), 505-509.

[3] L. Janos, H. M. Ko, and K. K. Tan, Edelstein's contractivity and attractors, Proc. Amer. Math. Soc. 76 (1979), no. 2, 339-344.

[4] Z. Liu, Fixed point theorems for condensing and compact maps, Kobe J. Math. 11 (1994), no. 1, 129-135.

[5] R. D. Nussbaum, Some asymptotic fixed point theorems, Trans. Amer. Math. Soc. 171 (1972), 349-375.

ZeQing LiU: DePartment of Mathematics, Liaoning Normal University, P.O. Box 200, DALIAN 116029, LIAONING, CHINA

E-mail address: zeqingliu@sina.com.cn

Li WANG: Department of Mathematics, Liaoning Normal University, P.O. Box 200, DALIAN 116029, LIAONING, CHINA

Shin Min Kang: Department of Mathematics, Gyeongsang National University, CHINJU 660-701, KOREA

E-mail address: smkang@nongae.gsnu.ac.kr

Yong Soo Kim: Department of Mathematics, GyeongSAng National University, CHINJU 660-701, KOREA 


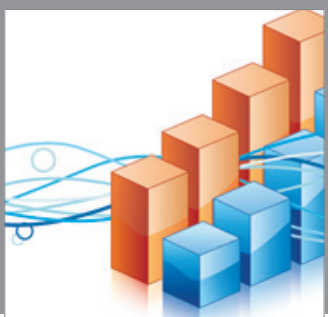

Advances in

Operations Research

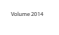

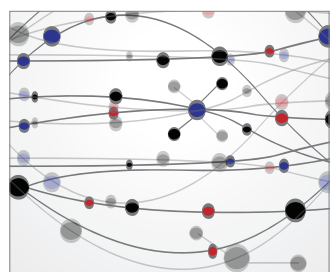

\section{The Scientific} World Journal
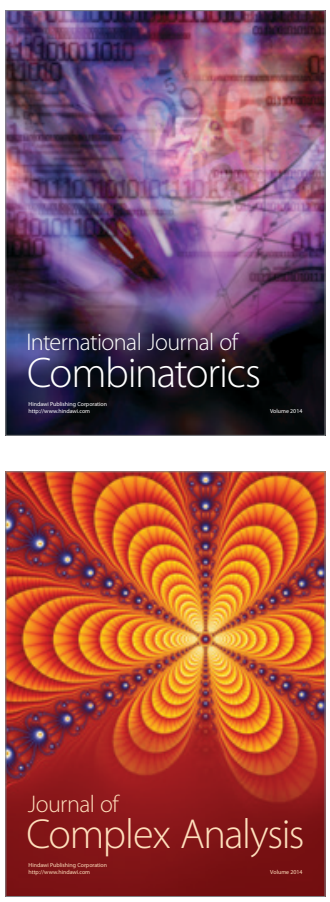

International Journal of

Mathematics and

Mathematical

Sciences
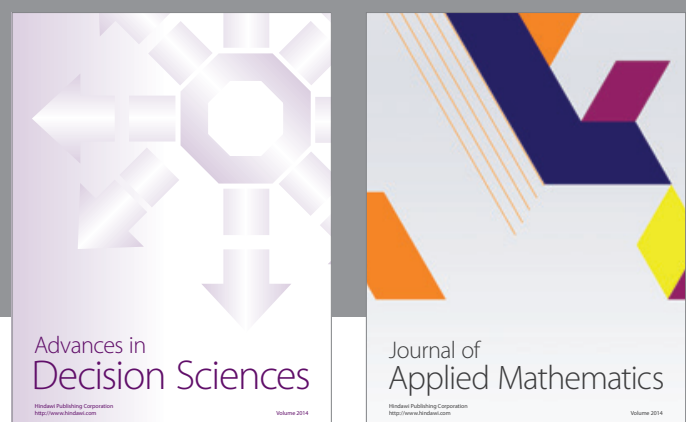

Journal of

Applied Mathematics
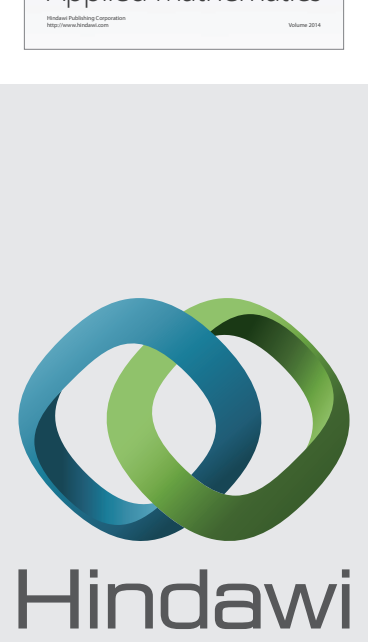

Submit your manuscripts at http://www.hindawi.com
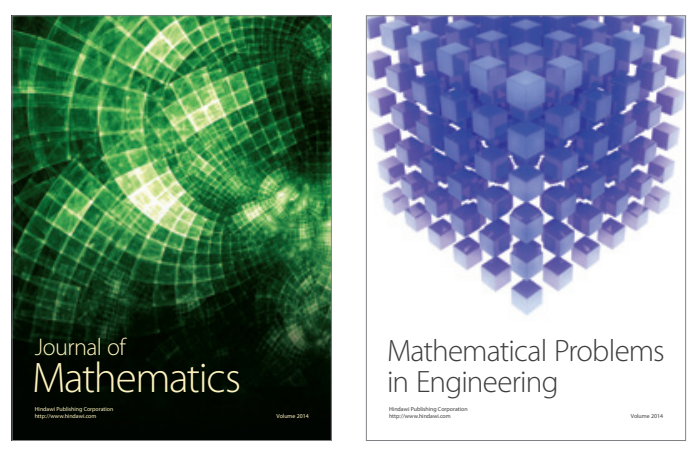

Mathematical Problems in Engineering
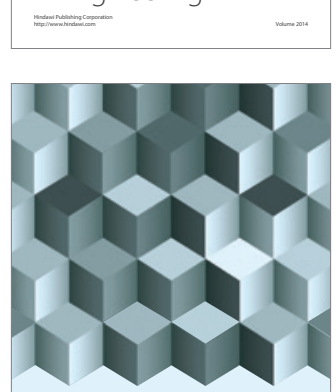

Journal of

Function Spaces
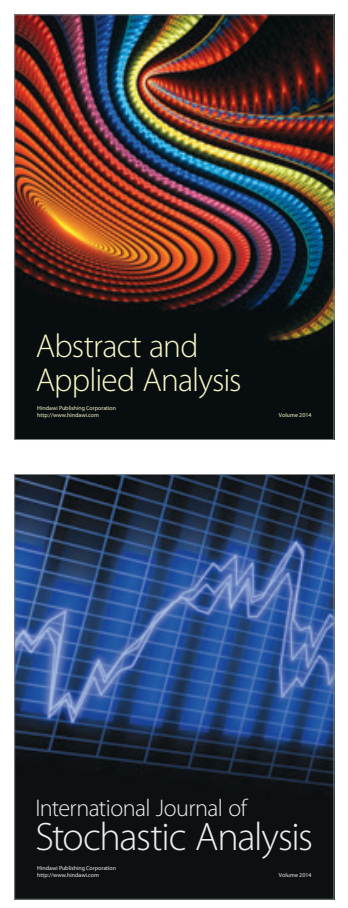

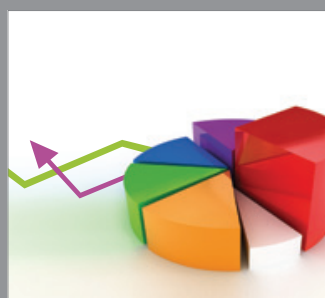

ournal of

Probability and Statistics

Promensencen
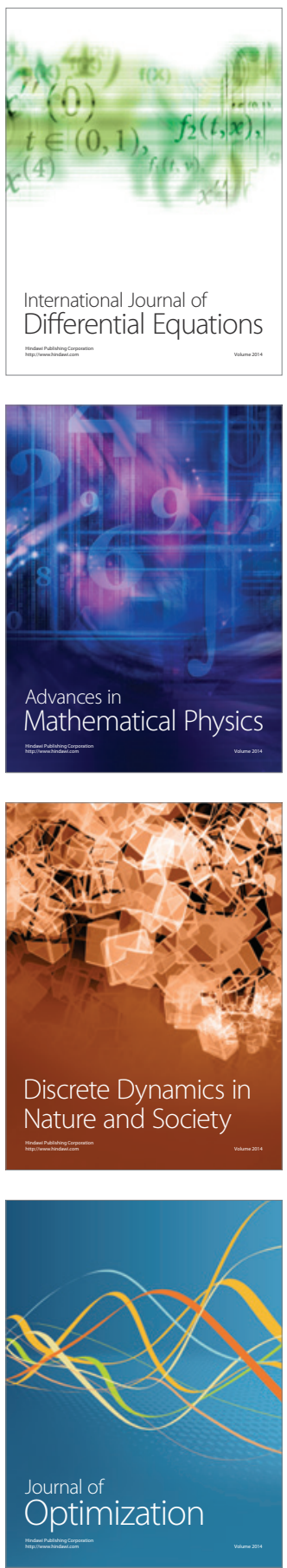